\title{
The Effects of Repeated Antenatal Glucocorticoid Therapy on the Developing Brain
}

\author{
NEENA MODI, HELEN LEWIS, NIRAN AL-NAQEEB, MORENIKE AJAYI-OBE, \\ CAROLINE J. DORÉ, AND MARY RUTHERFORD \\ Department of Pediatrics and Neonatal Medicine [N.M., N.A-N.] and Robert Steiner Magnetic Resonance \\ Unit [H.L., M.A-O., M.R.], Imperial College School of Medicine, Hammersmith Hospital, London \\ W12 0NN, UK, and Medical Research Council Clinical Trials Unit, London, UK [C.D.]
}

\begin{abstract}
ABST
Antenatal glucocorticoid (GC) therapy improves infant out-
come following preterm birth. As approximately $50 \%$ of women
given a first course of antenatal GCs remain undelivered 7-14 d
later, many clinicians administer further courses. GCs are known
to be neurotoxic and there is concern that exposure during early
development may have adverse effects on the immature brain.
The aim of this investigation was to compare magnetic resonance
(MR) indices of brain maturation in infants exposed to repeated
antenatal GC therapy and born at or close to term, with non-GC
exposed control infants. MR images were obtained during quiet
sleep without sedation. T1 weighted volume images were ob-
tained in the sagittal plane and T1, T2 weighted spin echo and
inversion recovery images in the transverse plane. Brain volume
and surface area were calculated from segmented image slices,
and a measure of the complexity of cortical folding, the whole
cortex convolution index (WCCI), from computerized analysis of
a vector coded contour following algorithm. Analysis of covari-
ance was used to compare the two groups after allowing for the
effect of postmenstrual age. There were 10 infants in the GC
group (range of antenatal GC exposure, 3 -11 courses) and 6
\end{abstract}
controls. Each GC course comprised two 12-mg IM doses of betamethasone $24 \mathrm{~h}$ apart. GC exposed infants had a significantly lower WCCI $(p=0.001)$ and smaller surface area $(p=0.02)$, after allowing for postmenstrual age. There was no significant difference in brain volume $(p=0.5)$. Repeated antenatal GC exposure results in measurable differences in brain maturation when compared with gestational age matched non-GC exposed controls. The clinical relevance of these observations is not known. (Pediatr Res 50: 581-585, 2001)

GC, glucocorticoid
MR, magnetic resonance
WCCI, whole cortex convolution index
OR, odds ratio
SE, spin echo
IR, inversion recovery
C $_{\text {SD }}$, SD of curvature
D $_{\mathbf{C}}$, cortical density

Randomized trials have demonstrated that antenatal glucocorticoid (GC) therapy accelerates fetal lung maturation and improves infant short term outcome following preterm birth by reducing respiratory distress syndrome (OR $0.53,95 \%$ confidence interval $=0.44,0.63)$, periventricular hemorrhage $(\mathrm{OR}$ $0.48,95 \% \mathrm{CI}=0.32,0.72)$, and neonatal death (OR 0.60, 95\% $\mathrm{CI}=0.48,0.75)(1)$. Maternal administration of GC steroids is now regarded as mandatory when the risk of preterm delivery is high.

Approximately $50 \%$ of women given a first course of antenatal GCs remain undelivered 7-14 d later. In this situation, many clinicians administer a further course or courses because

Received October 13, 2000; accepted March 24, 2001.

Correspondence: Dr. Neena Modi, Department of Pediatrics and Neonatal Medicine, Imperial College School of Medicine, Hammersmith Hospital, Du Cane Road, London W12 0NN, UK; e-mail: n.modi@ic.ac.uk

This work was supported by the Medical Research Council and Marconi Medical Systems. maximum benefit has been shown for babies delivered more than $24 \mathrm{~h}$ and less than $7 \mathrm{~d}$ after commencement of treatment. A recent survey of over 250 obstetric units in the UK has shown that the practice of administering repeated courses of antenatal GCs is widespread (2), although there is no evidence that this further improves neonatal outcome.

There is growing concern based on observations in vitro and in vivo, in animal models and in humans, that GCs are neurotoxic. There is also evidence that GC exposure during early development may adversely program the immature brain (3). The aim of this investigation was to compare magnetic resonance (MR) indices of brain maturation in infants who had been exposed to repeated antenatal GC therapy and who were born at or close to term, with non GC exposed controls.

\section{METHODS}

We approached mothers who had received multiple doses of antenatal GCs at intervals of one to two weeks, and who had 
delivered close to or at term, and mothers who had delivered full term infants and had not received antenatal steroids, for permission to enroll their infant into the study. The investigation was approved by the Research Ethics Committee of Hammersmith Hospitals Trust and Imperial College School of Medicine (formerly the Royal Postgraduate Medical School). Informed consent was obtained from all subjects and controls.

MR sequences and analyses. Infants were imaged in normal quiet sleep, as soon after delivery as possible. No sedation was used. Images were obtained in the sagittal plane using a T1 weighted volume acquisition (23/6). Additional transverse T1 (SE 860/20) and T2 (SE 2700/120) weighted spin echo and inversion recovery (IR3800/30/950) images were obtained in the transverse plane. All analyses were performed blinded to patient group.

Visual analyses. Images were assessed for normal anatomy and for the presence of any abnormalities.

Quantification analyses. A specifically designed computer program was used to measure the maturational folding of the cortex on the images $(4,5)$. An objective estimation of the complexity of cortical folding, the Whole Cortex Convolution Index (WCCI), cerebral tissue volume (brain volume excluding brainstem and cerebellum) and cortical surface area were measured from the T1 weighted volume acquisition sets. The cortex was segmented manually on each image slice to exclude bone and surrounding soft tissue (Fig. 1). Cerebral tissue volume for each slice was calculated as the product of the total number of voxels in the segmented brain image, dimensions of the voxels, and thickness of segmented brain slices. This

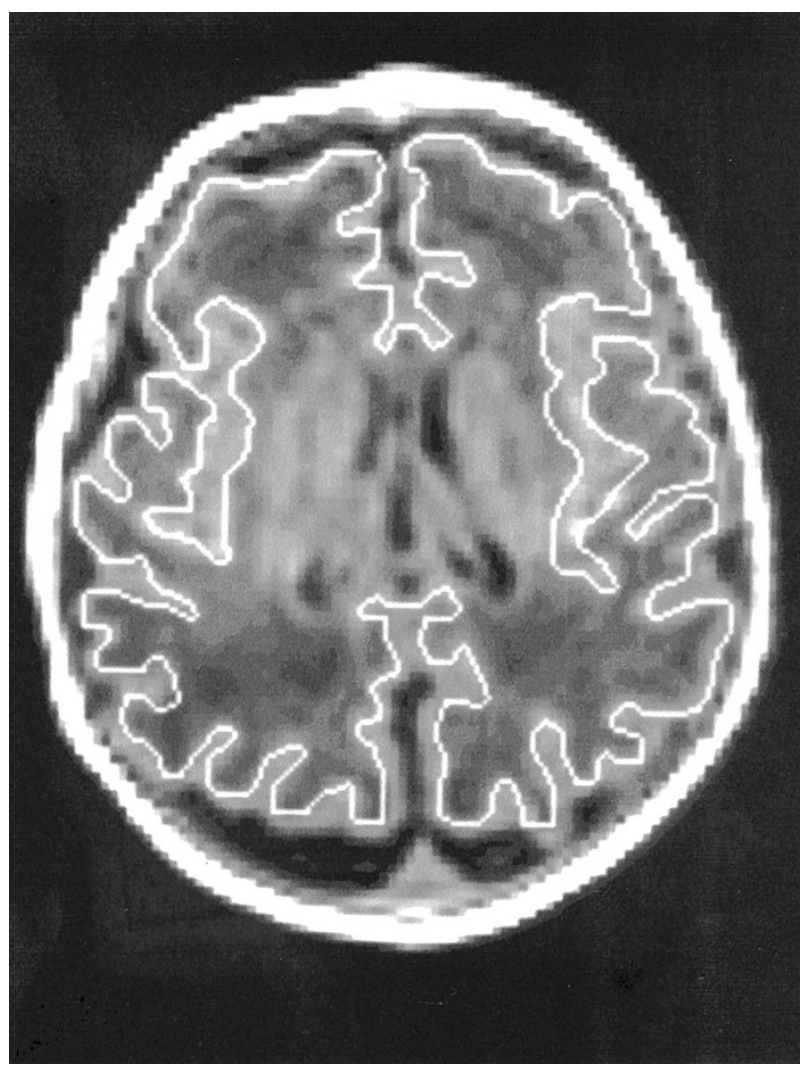

Figure 1. Transverse image of the brain showing cortical contour. measure included the lateral and third ventricles. The cortical surface area was calculated as the sum of the perimeter of voxels in each image slice multiplied by the thickness of the brain slices. To calculate the complexity of the cortical folding, the boundary of the segmented cortex was extracted using a contour following algorithm (4) and then coded in vector form using the Freeman 8-way chain code (4) to produce a curvature code. The latter gave a quantified description ranging from $-180^{\circ}$ to $+180^{\circ}$ in increments of $45^{\circ}$ for changes in orientation of adjacent contour vectors. The curvature code was smoothed using a Gaussian function to minimize effects of noise along the contour. The $\mathrm{SD}$ of curvature $\mathrm{C}_{\mathrm{SD}}$ for the whole segmented cortex in each slice was computed. To incorporate a measure of convolution depth, a dimensionless quantity that reflected cortical density, $\mathrm{D}_{\mathrm{C}}$, was obtained from the ratio of squared length of cortex to the area of the smallest rectangle enclosing the cortex boundary. The WCCI is the product of $\mathrm{C}_{\mathrm{SD}}$ and $\mathrm{D}_{\mathrm{C}}$. The mean WCCI for each brain was calculated from the values for each slice. Intra-observer and interobserver variability was less than $2 \%$.

Statistical analyses. Analysis of covariance was used to compare the two groups after allowing for the effect of postmenstrual age. The assumption of normality of residuals was checked using the Shapiro and Francia W' test and the assumption of parallel regression lines in the cases and controls using multiple regression.

\section{RESULTS}

There were 10 infants in the multiple antenatal GC group and six control infants. All infants were imaged within one week of birth. Three infants in the steroid group were the product of a triplet pregnancy. Four in the control group had a clinical history in keeping with mild birth asphyxia but did not develop neonatal encephalopathy and had normal imaging with no evidence of cerebral edema. They were subsequently shown to have a normal outcome and normal head growth. The two remaining control infants were recruited from the postnatal wards following an uneventful pregnancy and delivery. All mothers in the GC group had received betamethasone. The number of courses received ranged from 3 to 11. A "course" of treatment comprised two 12-mg doses given intramuscularly $24 \mathrm{~h}$ apart. The indications for GC therapy and the number of courses received are shown in Table 1.

Visual image analysis. All 16 infants showed normal brain anatomy. Two GC exposed infants had discrete lesions, a caudate cyst in one, and multiple small hemorrhagic white matter lesions (Fig. 2) in the second. Two GC exposed infants had some asymmetry of the lateral ventricles, but none of the infants showed abnormally dilated ventricles. Nine of the 10 GC exposed infants showed low signal intensity within the white matter on $\mathrm{T} 1$ weighted imaging and corresponding high signal intensity on the T2 weighted images. This was marked and involved both anterior and posterior white matter in six infants (Fig. 3) Two of the control infants had slightly low signal intensity within the white matter. None of the control group had discrete lesions or evidence of brain swelling. All 16 infants showed a normal appearance to the medial temporal 
Table 1. Indications for antenatal glucocorticoid therapy, type of steroid and number of courses

\begin{tabular}{|c|c|c|c|}
\hline Indication for antenatal glucocorticoid therapy & Type of steroid & $\begin{array}{c}\text { Number of courses } \\
(\text { each } 12 \mathrm{mg} \times 2)\end{array}$ & $\begin{array}{l}\text { Postmenstrual age at time } \\
\text { of imaging (weeks }+ \text { days) }\end{array}$ \\
\hline Triplet pregnancy & Betamethasone & 11 & $36+2$ \\
\hline Triplet pregnancy & Betamethasone & 11 & $36+2$ \\
\hline Prolonged preterm rupture of membranes & Betamethasone & 4 & $39+2$ \\
\hline Poor obstetric history & Betamethasone & 4 & $38+5$ \\
\hline Cervical incompetence with suture & Betamethasone & 3 & $38+3$ \\
\hline Prolonged preterm rupture of membranes & Betamethasone & 5 & $37+3$ \\
\hline Recurrent vaginal bleeding & Betamethasone & 4 & $35+1$ \\
\hline
\end{tabular}

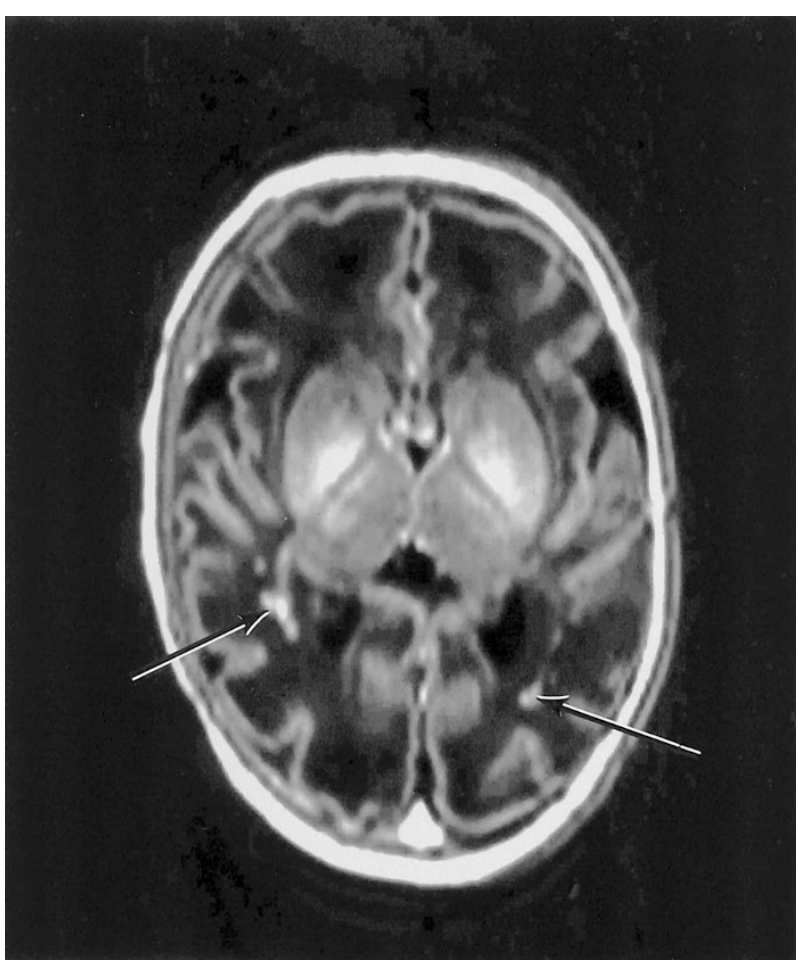

Figure 2. Inversion recovery (IR 3800/30/950) sequence in the transverse plane. Infant of $35 \mathrm{wk}$ gestation imaged at $2 \mathrm{~d}$ of age. There is generally low signal intensity within the white matter. There are discrete high signal intensity lesions (arrows) within the white matter consistent with hemorrhage. There is high signal intensity in the posterior putamen and globus pallidum and low signal intensity within the posterior limb of the internal capsule. This is normal for 35 wk gestation.

lobe, including the hippocampal region, and to the pituitary gland.

Quantitative image analysis. Analysis of covariance showed GC exposed infants to have a significantly lower WCCI $(p=0.001)$ and smaller surface area $(p=0.02)$, after allowing for postmenstrual age. There was no significant difference in brain volume between GC exposed infants and controls $(p=0.5)$ (Table 2).

\section{DISCUSSION}

Computer quantification of cortical folding is an objective analysis of brain maturation. It also enables the whole cortex to be assessed, rather than individual sulci and gyri, as is the case with visual analysis of maturation (6). We have shown using
MR quantification that newborn infants born close to term, who were exposed to multiple doses of antenatal GCs, show significant differences in cortical development when compared with control infants. Cortical folding, as assessed by the whole cortex convolution index (WCCI) and brain surface area, was reduced in the steroid exposed infants. Visual analysis of the MR images also showed a high incidence of long T1 and long T2. We did not detect any difference in brain volume. Quantification of the brain volume in these infants included the lateral and third ventricles, but as none of the infants had ventricular dilation, their contribution to total brain volume was accepted as constant.

We were unable to quantify the white matter changes in this group of infants. Measurement of the T1 and T2 values within specific areas of white matter would be more objective. However the changes demonstrated (Fig. 3) were considered abnormal and were detected by an observer unaware of whether the infant came from the GC treated or control group. A long T1 and long T2 suggest increased water content within the tissue. Body water content decreases with maturation. This observation, as well as the reduction in cortical folding, is consistent with the possibility that repeated antenatal GC exposure resulted in delayed brain maturation, but not in overall brain size.

The MR quantification indices may have been influenced by two factors other than GC exposure. Firstly, anticipated difficulties in recruiting healthy infants and then obtaining nonmotion artifacted images without the use of sedation, led to our decision to use images from four infants with a history of mild birth asphyxia. Infants with asphyxia may show brain swelling, with a decreased extracerebral space and sulcal narrowing that makes it difficult to segment the brain. However none of the four infants showed these changes and their head growth and neurodevelopmental outcome has proved to be normal, justifying their inclusion in the control group.

Secondly, three of the GC treated group were the result of a triplet pregnancy. Although such infants might be expected to have smaller growth, including a smaller brain size, than gestational aged matched singletons, and thus might have influenced the analyses, no difference between the groups was found in brain volume. Repeat data analysis after exclusion of the three triplet infants, did not alter our conclusions. This adds weight to our inference that GC exposure during early development affects brain maturation rather than overall brain volume. However, the possibility that repeated GC exposure may 


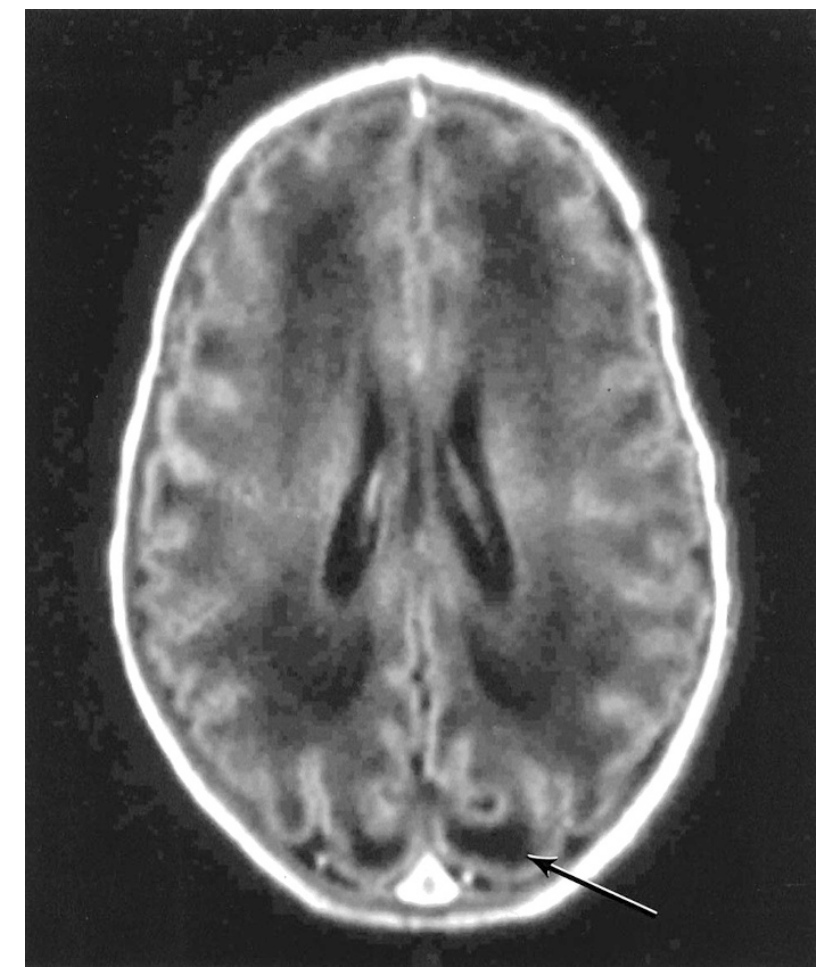

Figure 3. T1 weighted spin echo ( $\mathrm{SE} 860 / 20)$ sequences in one of triplets born at $35 \mathrm{wk}$ and imaged at $5 \mathrm{~d}$ of age. The white matter is generally low signal on T1 weighted images. There are more severe changes in regions of the subcortical white matter (arrow).

Table 2. Estimated means evaluated at a postmenstrual age of 37.8 weeks

\begin{tabular}{lccccc}
\hline & \multicolumn{2}{c}{ Steroid treated } & & \multicolumn{2}{c}{ Control } \\
\cline { 2 - 3 } \cline { 5 - 6 } & Mean & $95 \% \mathrm{CI}$ & & Mean & $95 \% \mathrm{CI}$ \\
\hline WCCI & 15.7 & $14.4,17.0$ & & 20.0 & $18.3,21.7$ \\
Surface area $\left(\mathrm{cm}^{2}\right)$ & 563 & 522,604 & & 649 & 594,703 \\
Volume $\left(\mathrm{cm}^{3}\right)$ & 457 & 424,490 & & 477 & \\
\hline
\end{tabular}

affect brain volume should not be discounted by our small study. Glucocorticoid steroids are initiators of apoptosis and thus might well influence organ growth. In the sheep fetus there is evidence of a dose-dependent reduction in brain weight and volume (7) and French et al. (8), in an observational study of 477 preterm singleton infants, demonstrated that multiple courses of antenatal steroids were associated with reduced fetal growth and head circumference corrected for gestational age. In the sheep, repeated doses of antenatal steroids disrupt optic nerve development myelination (9). Myelination has been affected after single dose glucocorticoid administration to newborn rats (10) and these authors also reported a reduction in total brain weight. There was no evidence of a reduction in myelination in our study, but there is very little new myelination within the immature brain between 25 and 35 wk gestation. Quantification of myelination at two years of age would be a more appropriate time to examine the effects of antenatal GCs.

In human adults, regions of the brain rich in GC receptors, such as the hippocampus, are particularly susceptible to steroid neurotoxicity. The hippocampus is involved in memory pro- cessing and in regulating neuroendocrine function and behavior. In a study designed to mimic clinical practice, maternal treatment with dexamethasone resulted in reduced hippocampal cell number and pronounced degeneration in the hippocampal CA3 region in fetal rhesus monkeys (11). Degeneration was dose dependent but occurred even with a dose of 0.5 $\mathrm{mg} / \mathrm{kg}$, which is comparable to the dose used to enhance fetal lung maturation in humans. Long term effects were studied in rhesus monkeys receiving dexamethasone $5 \mathrm{mg} / \mathrm{kg}$. At $20 \mathrm{mo}$ of age, magnetic resonance imaging of the brain showed an approximately $30 \%$ reduction in hippocampal volume in treated offspring, compared with control offspring (12). We did not quantify hippocampal volume as this is technically very difficult in the small unmyelinated brain. However visual analysis did not suggest hippocampal atrophy, and the appearances of the medial temporal lobe were normal.

In contrast to its effects on the mature brain, GC exposure during early development may also have more global effects that are likely to be related more to the stage of brain development and GC receptor expression rather than to whether exposure is in utero or after birth. However after birth, the very preterm infant is often exposed to multiple neuronal insults and may therefore be more susceptible than the unborn infant of similar gestation. Glucocorticoids impair neuronal ability to withstand excitotoxic and other neurotoxic insults, including hypoxia-ischemia and hypoglycemia, and thus may interact with and exacerbate other neurologic injury (13-15). Observations in extremely preterm human infants receiving GC therapy in the postnatal period, for the prevention and treatment of chronic lung disease of prematurity, point to an association with neurologic injury. The infants in these studies were at a comparable postmenstrual age and stage of brain development as infants exposed antenatally. In an open study in which two doses of $0.25 \mathrm{mg} / \mathrm{kg}$ dexamethasone were administered within $24 \mathrm{~h}$ of birth, Baud et al. (16) describe a doubling in the incidence of periventricular leukomalacia, from $7 \%$ before and after the institution of this treatment regimen to $15 \%$ during the period of intervention. Two recent randomized double blind, placebo controlled trials evaluating dexamethasone therapy to decrease the risk and severity of chronic lung disease have demonstrated an increase in neuromotor dysfunction at 2 y (17) and in cerebral palsy (18). Two infants in our study showed focal lesions, one a caudate cyst consistent with an antenatal distal middle cerebral artery infarction, and the other multiple small hemorrhagic lesions within the white matter. Neither of these lesions can necessarily be attributed to GC therapy nor would they be associated with the development of cerebral palsy.

Infants born preterm are already at substantial risk of adverse neurodevelopmental outcome. By reducing acute disease severity, a single course of antenatal GC therapy improves survival and reduces periventricular hemorrhage, an important determinant of long-term outcome. These powerful effects may mask more subtle, adverse long-term consequences. MacArthur et al. (19) described impaired visual memory at the age of $6 \mathrm{y}$ in children exposed antenatally to betamethasone, although noting that there was no overall neurologic impairment. There have been no human studies addressing the impact 
Table 3. Postmenstrual age at time of study, WCCI, brain surface area and volume: cases and controls

\begin{tabular}{ccccc}
\hline & $\begin{array}{c}\text { Postmenstrual age } \\
\text { (weeks }+ \text { days) }\end{array}$ & WCCI & $\begin{array}{c}\text { Surface area } \\
\left(\mathrm{cm}^{2}\right)\end{array}$ & $\begin{array}{c}\text { Volume } \\
\left(\mathrm{cm}^{3}\right)\end{array}$ \\
\hline Infant (cases) & & & & \\
1 & $36+2$ & 12.92 & 488.42 & 477.99 \\
2 & $38+5$ & 15.71 & 580.78 & 419.97 \\
3 & $39+2$ & 18.04 & 650.76 & 511.70 \\
4 & $36+2$ & 13.96 & 538.15 & 431.55 \\
5 & $38+3$ & 18.79 & 584.86 & 437.96 \\
6 & $36+2$ & 14.79 & 533.57 & 458.55 \\
7 & $34+4$ & 9.48 & 392.75 & 392.97 \\
8 & $36+4$ & 11.22 & 446.19 & 425.10 \\
9 & $37+3$ & 15.68 & 616.06 & 499.92 \\
10 & $35+1$ & 9.35 & 377.03 & 364.89 \\
Infants (controls) & & & & \\
1 & 38 & 20.21 & 709.02 & 515.98 \\
2 & $41+5$ & 24.24 & 738.93 & 493.87 \\
3 & $41+3$ & 28.82 & 908.11 & 594.06 \\
4 & 42 & 31.01 & 840.14 & 513.44 \\
5 & 35 & 14.12 & 459.45 & 369.95 \\
6 & 37 & 18.77 & 660.96 & 519.56 \\
\hline
\end{tabular}

of repeated antenatal GC exposure on later neurodevelopmental outcome in preterm babies nor on outcome for babies who go on to be delivered at term.

Present evidence with regard to postnatal GC therapy for chronic lung disease in preterm infants suggests that a "therapeutic window" exists between 7-10 d after birth (20). During this time, the benefits of treatment outweigh the adverse effects. The role of a single course of antenatal GC therapy in enhancing lung maturation and improving outcome after preterm birth is one of the successes of present day perinatal practice. However, when the patient continues at high risk of preterm delivery, the optimum strategy is unknown. In this situation, repeated courses of antenatal therapy may have benefits that outweigh any adverse effects. Conversely, an equally plausible hypothesis is that the adverse effects of multiple courses outweigh the benefits of a single course. Our study suggests that brain maturation is delayed, although the clinical relevance of this is uncertain. Other evidence also suggests that caution is warranted. For the present, multiple courses of antenatal GCS are best confined to randomized trials incorporating neuroimaging and detailed long-term neurodevelopmental surveillance.

Acknowledgments. Neena Modi had the original idea for this study. Niran Al-Naqeeb recruited subjects and assisted in imaging. Mary Rutherford, Helen Lewis, and Morenike AjayiObe analyzed the images. Caroline Doré and Neena Modi analyzed the results. The paper was written by Mary Rutherford and Neena Modi.

Nadeem Saeed wrote the computer program for quantifying the cortical folding.

\section{REFERENCES}

1. Crowley P 1999 Prophylactic corticosteroids for preterm delivery. (Cochrane Review). In: The Cochrane Library, Issue2 Oxford: Update Software

2. Brocklehurst P, Gates S, McKenzie-McHarg K, Alfirevic Z, Chamberlain G 1999 Are we prescribing multiple courses of antenatal corticosteroids? A survey of practice in the UK. Br J Obstet Gynaecol 106:977-979

3. Matthews SG 2000 Antenatal glucocorticoids and programming of the developing CNS. Pediatr Res 47:291-300

4. Saeed N, Ajayi-Obe M, Counsell S, Hajnal J, Rutherford MA 1998 Convolution index computation of the cortex using image segmentation and contour following. International Society Magnetic Resonance of Medicine, Abstract 2076

5. Ajayi-Obe M, Saeed N, Cowan FM, Rutherford MA, Edwards AD 2000 Reduced development of cerebral cortex in extremely preterm infants. Lancet 30:1162-1163

6. Battin MR, Maalouf EF, Counsell SJ, Herlihy AH, Rutherford MA, Azzopardi D, Edwards AD 1998 Magnetic resonance imaging of the brain in preterm infants: visualization of the germinal matrix, early myelination and cortical folding. Pediatrics 101:957-962

7. Huang WL, Beazley LD, Quinlivan JA, Evans SF, Newnham JP, Dunlop SA 1999 Effect of corticosteroids on brain growth in fetal sheep. Obstet Gynaecol 94:213-218

8. French NP, Hagan R, Evans SF, Godfrey M, Newnham J 1999 Repeated antenatal corticosteroids: size at birth and subsequent development. Am J Obstet Gynaecol 180:114-121

9. Dunlop S, Archer M, Quinlivan J, Beazley L, Newnham J 1997 Repeated prenatal corticosteroids delay myelination in the ovine central nervous system. J Matern Fetal Med 6:309-313

10. Gumbinas M, Oda M, Huttenlocher P 1973 The effect of corticosteroids on myelination of the developing rat brain. Biol Neonate 22:355-366

11. Uno H, Lohmiller L, Thieme C, Kemnitz JW, Engle MJ, Roecker EB, Farrel PM 1990 Brain damage induced by prenatal exposure to dexamethasone in fetal rhesus macaques. 1. Hippocampus. Dev Brain Res 53:157-167

12. Uno H, Eisele S, Sakai A, Shelton S, Baker E, DeJesus O, Holden J 1994 Neurotoxicity of glucocorticoids in the primate brain. Horm Behav 28:336-348

13. Tombaugh GC, Yang SH, Swanson RA, Sapolsky RM 1992 Glucocorticoids exacerbate hypoxic and hypoglycaemic hippocampal injury in vitro: biochemical correlates and a role for astrocytes. J Neurochem 59:137-146

14. Stein-Behrens BA, Lin WJ, Sapolsky RM 1994 Physiological elevations of glucocorticoids potentiate glutamate accumulation in the hippocampus. J Neurochem 63:596602

15. Moghaddam B, Bolinao ML, Stein-Behrens B, Sapolsky R 1994 Glucocorticoids mediate the stress induced extracellular accumulation of glutamate. Brain Res 655:251-254

16. Baud O, Zupan V, Lacaze-Masmonteil T, Dehan M 1999 Neurological adverse effects of early postnatal dexamethasone in very preterm infants. Arch Dis Child 80:F159

17. Yeh TF, Lin YJ, Huang CC, Chen YJ, Lin CH, Hsieh WS, Lien YJ 1998 Early dexamethasone therapy in preterm infants: a follow up study. Pediatrics 101(5):E7

18. O'Shea TM, Kothadia JM, Klinepeter KL, Goldstein DJ, Jackson BG, Weaver RG, Dillard RG 1999 Randomized placebo-controlled trial of a 42-day tapering course of dexamethasone to reduce the duration of ventilator dependency in very low birth weight infants: outcome of study participants at 1-year adjusted age. Pediatrics 104:15-21

19. MacArthur BA, Howie RN, Dezoete JA, Elliott DA 1982 School progress and cognitive development of 6 year old children whose mothers were treated antenatally with betamethasone. Pediatrics 70:99-105

20. Tarnow-Mordi W, Mitra A 1999 Postnatal dexamethasone in preterm infants. BMJ 319:1385-1386 